\title{
High-Sensitivity C-Reactive Protein in Metabolic Healthy Obesity (MHO)
}

\author{
Aamil Rasheed ${ }^{1}$, Sourya Acharya ${ }^{2}$, Samarth Shukla 3 , Sunil Kumar4, Roopesh Yarappa ${ }^{5}$, Yash Gupte ${ }^{6}$, \\ Vidyashree Hulkoti ${ }^{7}$
}

\begin{abstract}
${ }^{1}$ Department of Medicine, DMIMS (Deemed to be University) Sawangi (M), Wardha, Maharashtra, India. ${ }^{2}$ Department of Medicine, DMIMS (Deemed to be University) Sawangi (M), Wardha, Maharashtra, India. ${ }^{3}$ Department of Pathology, DMIMS (Deemed to be University) Sawangi (M), Wardha, Maharashtra, India. ${ }^{4}$ Department of Medicine, DMIMS (Deemed to be University) Sawangi (M), Wardha, Maharashtra, India. ${ }^{5}$ Department of Medicine, DMIMS (Deemed to be University) Sawangi (M), Wardha, Maharashtra, India. ${ }^{6}$ Department of Medicine, DMIMS (Deemed to be University) Sawangi (M), Wardha, Maharashtra, India. ${ }^{7}$ Department of Medicine, DMIMS (Deemed to be University) Sawangi (M), Wardha, Maharashtra, India.
\end{abstract}

\section{ABSTRACT}

\section{BACKGROUND}

Metabolically Healthy Obesity/Metabolic Healthy Obesity (MHO) is a paradox in scientific medical literature and discussion is still on regarding the safety status of MHO phenotype. It is an obesity phenotype where the subjects have BMI more than or equal to $30 \mathrm{Kg} / \mathrm{m}^{2}$ but are devoid of conventional metabolic complications such deranged lipid profile, altered glucose tolerance, or metabolic syndrome as they have less adverse inflammatory profile, low visceral fat, less disturbed insulin signalling, and lipid metabolism. But recently studies are coming up with robust evidence that $\mathrm{MHO}$ is not a benign condition. It may lead to metabolic syndrome in future and it is also associated with cardiometabolic risks.

\section{METHODS}

This cross-sectional study was done in a tertiary care hospital conducted for a period of two years from October 2017 to October 2019. After obtaining institutional ethical clearance, this cross-sectional study was conducted on 120 MHO subjects, 120 metabolic syndrome (MS) and 120 Metabolic Healthy Non-Obese (MHNO) subjects. Anthropometric data was obtained, and hs-CRP was estimated and compared with MS and MHNO group. The data was analysed using appropriate statistical significance tests.

\section{RESULTS}

In one-way Analysis of Variance (ANOVA), anthropometric determinants and metabolic variables differed significantly across the groups $(\mathrm{p}<0.0001)$. The mean hsCRP in MHO was; $4.45 \pm 1.46$ and in the control group it was $1.84 \pm 0.77(\mathrm{p}<0.0001)$. Using Pearson's correlation coefficient, significant positive correlation was found between hs-CRP with other anthropometric and metabolic parameters. In multiple regression analysis, Body Mass Index (BMI), Waist Circumference (WC), were significantly associated with elevated hs-CRP. Adjusted odd's (AOR) of abnormal hsCRP in MHO was 1.9 times that of MHNO subjects.

\section{CONCLUSIONS}

MHO phenotype is associated with increased hs-CRP levels as compared to MHNO phenotype suggesting that obesity even if associated with a healthy metabolic profile, still harbour subclinical inflammation. So, subjects with MHO should be targeted for appropriate preventive strategies in the form of health education, lifestyle alterations to avoid future cardiovascular morbidities. MHO phenotype with evidence of subclinical vascular inflammation should not be considered a benign condition.

\section{KEY WORDS}

High Sensitivity C-Reactive Protein, Metabolic Syndrome, Metabolic Healthy NonObese

\author{
Corresponding Author: \\ Dr. Sourya Acharya, \\ Department of Medicine, \\ DMIMS (Deemed to be University) \\ Sawangi (M), Wardha, Maharashtra, India. \\ E-mail: souryaacharya74@gmail.com
}

DOI: $10.14260 /$ jemds/2020/100

Financial or Other Competing Interests: Dr. Acharya Reports Grants from DMIMS University, outside the submitted work.

How to Cite This Article:

Rasheed A, Acharya S, Shukla S, et al. Highsensitivity c-reactive protein in (MHO). J. Evolution Med. Dent. Sci. 2020;9 (07):443447, DOI: 10.14260/jemds/2020/100

Submission 27-11-2019,

Peer Review 22-01-2020,

Acceptance 29-01-2020,

Published 17-02-2020.

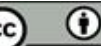




\section{BACKGROUND}

Metabolically healthy obese/Metabolic healthy obesity (MHO) is an obesity phenotype where the subjects have BMI more or equal to $30 \mathrm{Kg} / \mathrm{m}^{2}$ but are devoid of metabolic complication such as deranged lipid profile, altered glucose tolerance, or metabolic syndrome. Metabolically healthy obese have a less adverse inflammatory profile, low visceral fat, fewer intrusion of macrophages into adipose tissue, and minor fat cell size, not as much of disturbed, insulin signalling, and lipid metabolism, which may make them more responsive to dietary interventions. ${ }^{[1-5]}$ The estimated prevalence of MHO varies from 6 to 75 percent. [6] Available literature suggest that 10 to $25 \%$ percent of obese individuals have a healthy metabolic status and is more common in females and its prevalence decreases with age. ${ }^{[7,8]} \mathrm{MHO}$ the term safeguards obesity with evidence of absent cardiometabolic risk factors. But contrary to the belief researches are now providing ample evidence that MHO individuals are at an increased risk of developing type II diabetes mellitus and adverse cardiovascular events.[6] A individual's health status can switch from metabolically healthy obesity to metabolically unhealthy and vice versa. An important question is whether development of the MHO phenotype is time-dependent, as many studies has shown that nearly half of initially metabolically healthy obese subjects, shifted to the metabolically unhealthy phenotype after 10 years, with similar proportions in overweight and obese subjects.[5]

Subclinical disease assessments are becoming increasingly important as they provide an overview of the actual course of disease and more reliable prediction of events than traditional risk factors for CVD. Studies have proved that Highsensitivity/High sensitivity/highly sensitive CRP (hs-CRP) is an established marker of cardiovascular disease. This circulating pro-inflammatory cytokine leads to inflammation of the coronary arteries leading to cardiovascular disease. [7] Very limited research work has been done till date focusing different parameters of subclinical cardiovascular risk profiles apart from dyslipidaemia and diabetes in MHO populations. As already researches are gaining substantial data regarding existing cardiovascular risk in MHO population, this study will interestingly add to the existing data simultaneously incorporating one of subclinical cardiovascular risk marker, High-sensitivity C-reactive protein (hs-CRP) levels and their association with MHO population.

We wanted to estimate hs-CRP levels in MHO subjects, compare hs-CRP levels of MHO population with metabolic syndrome and metabolic healthy non obese (MHNO) subjects and correlate the clinical data (age, gender, blood pressure) and traditional metabolic syndrome variables (BMI, WC, FBS, HDL, TG) with hs-CRP in MHO population. High sensitivity Creactive protein (hs-CRP) is a well-established marker of vascular/endothelial inflammation, and studies have confirmed that increased hs-CRP increases risk of cardiovascular disease (CVD).

\section{METHODS}

This cross-sectional study with comparison group entitled "High sensitivity CRP in MHO subjects" was carried out over a period of two years (October 2017 to October 2019) in a tertiary care hospital. Institutional ethical committee (DMIMSU), clearance was taken before starting the study.

All cases were randomly selected from the university students, staff, workers, and various health check-up camps organised by the hospital. Relevant demographic data (information comprised of gender, age, occupation and postal address) was collected after taking due consent. History of Diabetes mellitus, systemic hypertension was taken. Detailed drug history was obtained. Subjects with infections, sepsis, coronary artery disease, chronic liver and kidney disease, rheumatologic disorders, alcoholics, women on contraceptive pills, and subjects not giving consent were excluded. Metabolic unhealthy with normal weight phenotypes were also excluded. Detailed physical examination and anthropometric measurement in form of BMI, WC was calculated. Biochemistry analysis including FBS, TG, HDL-C was estimated.

Obesity in this study was defined as per the Body Mass Index (BMI) categories for Asian Indians that has been revised based on consensus guidelines. The revised guidelines categorise obesity as a BMI $\geq 25 \mathrm{Kg} / \mathrm{m}^{2}{ }^{2}\left[{ }^{[9]}\right.$ MS was defined as per the Modified National cholesterol education programme adult treatment panel III (NCEP ATP III) criteria as proposed by the AHA/NHLB [9,10]

1. Abdominal obesity (waist circumference $\geq 90 \mathrm{~cm}$ for Asian men or $\geq 80 \mathrm{~cm}$ for Asian women),

2. Triglycerides $\geq 150 \mathrm{mg} / \mathrm{dL}$

3. HDL cholesterol $\leq 40 \mathrm{mg} / \mathrm{dL}$ for men or $50 \mathrm{mg} / \mathrm{dL}$ for women

4. Systolic/diastolic blood pressure $\geq 130 / 85 \mathrm{mmHg}$ or receiving drug treatment

5. Fasting plasma glucose $\geq 100 \mathrm{mg} / \mathrm{dL}$.

MHO was defined as subjects with; $\mathrm{BMI} \geq 25 \mathrm{Kg} / \mathrm{m}^{2}$ (as per BMI category for Asians) with less than 3 MS criteria as per revised NCEP ATP III guidelines. ${ }^{[9,11]}$ In this study MHNO controls were defined as: $\mathrm{BMI} \leq 25 \mathrm{Kg} / \mathrm{m}^{2}$ with less than $3 \mathrm{MS}$ variables.[11] After taking due consent from the participants, Serum hs-CRP, Anthropometric data including weight, height, BMI, WC was measured by standard methods.[12] Blood Pressures (BP) was measured as per standard protocol.[13] Subjects with BP $\geq 130 / 85 \mathrm{~mm}$ of $\mathrm{Hg}$ or with ongoing treatment for hypertension was included as a parameter for defining MS. Fasting plasma glucose was estimated by the Glucose Oxidase (GOD)/Peroxidase (POD) method, serum HDL by direct enzymatic method, TG were estimated using a LIQUID STABLE GPO-PAP method by machine Robonic SemiAutomatic Chemical Analyser. Quantitative hs-CRP was estimated by a solid phase ultra-sensitive enzyme immunoassay based on two-site sandwich enzyme immunoassay technique. A serum hs-CRP levels $<1,1-3$ and $>3$ $\mathrm{mg} / \mathrm{L}$ are taken as low, intermediate, and high-risk groups for CVD risk as per guidelines. This study considered hs-CRP value $\geq 3 \mathrm{mg} / \mathrm{L}$ as abnormal reflecting sub clinical vascular inflammation.[14]

\section{Sample Size}

As per a large Asian observational cross-sectional study the proportion of patients with subclinical carotid atherosclerosis of metabolically healthy (MHO) and metabolically unhealthy (MUO) patients were $31.2 \%$ and $43.5 \%$ respectively.[15] formula for calculating the sample size is as follows- 


$$
\mathrm{n}=\left(\mathrm{Z}_{\alpha / 2}+\mathrm{Z}_{\beta}\right)^{2 *}\left(\mathrm{p}_{1}\left(1-\mathrm{p}_{1}\right)+\mathrm{p}_{2}\left(1-\mathrm{p}_{2}\right)\right) /\left(\mathrm{p}_{1}-\mathrm{p}_{2}\right)^{2}
$$

where, $\mathrm{Z}_{\alpha / 2}$ is the critical value of the Normal distribution at $\alpha / 2$ (e.g. for a confidence level of $95 \%, \alpha$ is 0.05 and the critical value is 1.96), $Z_{\beta}$ is the critical value of the Normal distribution at $\beta$ (e.g. for a power of $80 \%, \beta$ is 0.2 and the critical value is 0.84 ) and $\mathrm{p}_{1}$ and $\mathrm{p}_{2}$ are the expected sample proportions of the two groups. Here $\mathrm{p} 1=0.312$ and $\mathrm{p} 2=0.435$. Here $\mathrm{p} 1=0.312$ and $\mathrm{p} 2=0.435, \mathrm{Z}_{\alpha / 2}=1.96 \mathrm{Z}_{\beta}=0.8416$, $\left(\mathrm{Z}_{\alpha / 2}+\mathrm{Z}_{\beta}\right)^{2}=7.84, \mathrm{p} 1(1-\mathrm{p} 1)+\mathrm{p} 2(1-\mathrm{p} 2)=0.4604,\left(\mathrm{Z}_{\alpha / 2}+\mathrm{Z}_{\beta}\right)^{2}$ * $\left(\mathrm{p}_{1}\left(1-\mathrm{p}_{1}\right)+\mathrm{p}_{2}\left(1-\mathrm{p}_{2}\right)\right)=3.60,(\mathrm{p} 1-\mathrm{p} 2)^{2}=0.015129, \mathrm{n}=$ $3.60 / 0.015129=237.95 \sim 230$.

Thus, there will be need of total 230 study patients with $80 \%$ power at $95 \%$ confidence level. The number of patients in each group in the ratio $1: 1$ would be; $230 / 2=115$. Thus, the required sample size for each group will be 115 . To account for the calculated sample size; 120 subjects were analysed in MS, MHO and MHNO group each in a 1:1:1 ratio.

\section{Statistical Analysis}

The quantitative data were expressed as mean \pm SD. Pearson's correlation coefficient was used to test correlations between variables. Analysis of Variance Tests (ANOVA) and chi-square tests of independence were used for continuous and categorical variables, respectively. Logistic regression analysis was performed, adjusting for age, gender, WC, hypertension, FBS, LDL, HDL, and TG. A p-value $<0.05$ was considered to be statistically significant.

\section{RESULTS}

120 MHO, 120 MS and 120 MHNO subjects were included in the study. The MHO group was younger $(34.46 \pm 18.54)$ as compared to MS group $(52.20 \pm 16.58)$ and control group (42.35 \pm 14.5$)$, and proportion of females were more in MHO group (66\%). The male to female ratio in MHO group was 1:1.22. Studies have supported the view that MHO usually has a female preponderance. ${ }^{[7,8]} \mathrm{MHO}$ individuals were younger than the referent population. All the traditional variables and anthropometric determinants of metabolic syndrome (WC, SBP, DBP) and the parameters of metabolic syndrome (HDL, FBS, TG) differed significantly across the groups. Metabolic syndrome had the higher risk values followed by MHO and lastly the control group. The subclinical risk parameter hs CRP, showed similar trends across the groups which were statistically significant $(\mathrm{p}<0.05)$.

\section{Descriptive Statistics}

Mean hs-CRP level in MS subjects was $5.22 \pm 1.88$, in MHO subjects it was and in control subjects it was $4.45 \pm 1.46$. By using one-way ANOVA statistically significant variation was found in mean hs-CRP level among three groups of subjects ( $\mathrm{F}=75.86, \mathrm{p}=0.0001)$. On comparing mean hs-CRP level in three groups using Multiple Comparison: Tukey Test statistically significant difference was found between MS and MHO subjects $(\mathrm{p}=0.002)$ and between MHO and control subjects $(\mathrm{p}=0.0001)$. [Table 2].
When proportion and Odd's ratios for hs-CRP across weight and metabolic risk factor-based phenotype was assessed, the proportion of abnormal hs-CRP in MHO was $68.33 \%$. The adjusted odd's (AOR) of abnormal hs-CRP in MHO was 1.9 times that of MHNO subjects. In males it was 1.5 and in females it was 1.6 times more than MHNO subjects.

\begin{tabular}{|c|c|c|c|c|}
\hline Characteristics & MS & MHO & Control & $\mathbf{p}$ \\
\hline $\mathbf{N}$ & 120 & 120 & 120 & \\
\hline Age (yrs.) & $\begin{array}{c}52.20 \pm 16.58 \\
(20-75)\end{array}$ & $\begin{array}{c}34.46 \pm 18.54 \\
(18-66)\end{array}$ & $\begin{array}{c}42.35 \pm 14.50 \\
(18-70)\end{array}$ & $0.0001, \mathrm{~S}$ \\
\hline \multicolumn{5}{|c|}{ Gender } \\
\hline Male & 60 & $54(45 \%)$ & 60 & \multirow{4}{*}{$0.011, \mathrm{~S}$} \\
\hline & & & & \\
\hline Female & 60 & $66(55 \%)$ & 60 & \\
\hline M:F Ratio & $1: 1$ & $1: 1.22$ & $1: 1$ & \\
\hline BMI & $\begin{array}{c}30.72 \pm 4.60 \\
20.60-42.10)\end{array}$ & $\begin{array}{c}28.45 \pm 4.20 \\
(25-42.50)\end{array}$ & $\begin{array}{c}20.46 \pm 1.54 \\
(15-24.65)\end{array}$ & $0.0001, \mathrm{~S}$ \\
\hline \multicolumn{5}{|c|}{ Waist Circumference } \\
\hline Overall & $\begin{array}{c}102.00 \pm 14.18 \\
(74-130)\end{array}$ & \begin{tabular}{|c|}
$93.14 \pm 15.48$ \\
$(74-126)$
\end{tabular} & $\begin{array}{c}76.76 \pm 6.40 \\
(68-90)\end{array}$ & $0.0001, \mathrm{~S}$ \\
\hline Males & $\begin{array}{c}96.70 \pm 10.43 \\
(74-130)\end{array}$ & $\begin{array}{c}94.45 \pm 16.26 \\
(74-126)\end{array}$ & $\begin{array}{c}74.78 \pm 8.62 \\
(68-90)\end{array}$ & $0.0001, \mathrm{~S}$ \\
\hline Females & $\begin{array}{c}89.31 \pm 10.81 \\
(64-120)\end{array}$ & $\begin{array}{c}90.46 \pm 9.75 \\
(70-120)\end{array}$ & $\begin{array}{c}77 \pm 6.78 \\
(68-88)\end{array}$ & $0.0001, S$ \\
\hline \multicolumn{5}{|c|}{ BP } \\
\hline SBP & $\begin{array}{c}136 \pm 12.40 \\
(110-178)\end{array}$ & $\begin{array}{c}126.41 \pm 10.66 \\
(100-162)\end{array}$ & $\begin{array}{c}121.55 \pm 10.20 \\
(100-140)\end{array}$ & $0.0001, \mathrm{~S}$ \\
\hline DBP & $\begin{array}{c}90.66 \pm 10.45 \\
(70-110)\end{array}$ & $\begin{array}{c}86.66 \pm 6.72 \\
(70-104)\end{array}$ & $\begin{array}{c}72.56 \pm 8.66 \\
(60-90)\end{array}$ & $0.0001, \mathrm{~S}$ \\
\hline FBS & & & & \\
\hline FBS & $\begin{array}{c}95.56 \pm 16.81 \\
(60-135)\end{array}$ & $\begin{array}{c}92.56 \pm 16.56 \\
(59-240)\end{array}$ & $\begin{array}{c}84.66 \pm 12.25 \\
(38-100)\end{array}$ & $0.0001, \mathrm{~S}$ \\
\hline \multicolumn{5}{|c|}{ HDL } \\
\hline Overall & $\begin{array}{c}33 \pm 7.56 \\
(26-64)\end{array}$ & $\begin{array}{c}34.75 \pm 12.21 \\
(14-74)\end{array}$ & $\begin{array}{c}40.60 \pm 9.40 \\
(20-55)\end{array}$ & $0.004, S$ \\
\hline Males & $\begin{array}{c}32.44 \pm 10.02 \\
(26-64)\end{array}$ & $\begin{array}{c}33.82 \pm 11.41 \\
(14-65)\end{array}$ & $\begin{array}{c}37.46 \pm 7.04 \\
(18-55)\end{array}$ & $0.006, \mathrm{~S}$ \\
\hline Females & $\begin{array}{c}34.48 \pm 8.46 \\
(24-55)\end{array}$ & $\begin{array}{c}35.62 \pm 10.65 \\
(20-74)\end{array}$ & $\begin{array}{c}44.88 \pm 10.56 \\
(27-50)\end{array}$ & $0.001, \mathrm{~S}$ \\
\hline \multicolumn{5}{|c|}{ Triglyceride } \\
\hline TG & $\begin{array}{c}180.80 \pm 80.55 \\
(66-580)\end{array}$ & $\begin{array}{c}130.44 \pm 40.22 \\
(58-278)\end{array}$ & $\begin{array}{c}122.36 \pm 36.21 \\
(51-360)\end{array}$ & $0.0001, \mathrm{~S}$ \\
\hline \multicolumn{5}{|c|}{ Hs-CRP } \\
\hline hs CRP & $\begin{array}{l}5.22 \pm 1.88 \\
(1.20-8.60)\end{array}$ & $\begin{array}{l}4.45 \pm 1.46 \\
(0.80-9.50)\end{array}$ & $\begin{array}{l}1.84 \pm 0.77 \\
(1.00-3.20)\end{array}$ & $0.0001, \mathrm{~S}$ \\
\hline \multicolumn{5}{|c|}{ Table 1. Baseline Characteristics of Study Participants } \\
\hline
\end{tabular}

\begin{tabular}{|c|c|c|c|c|c|c|c|c|}
\hline \multirow[t]{2}{*}{ 苛 } & \multirow[t]{2}{*}{ z } & \multirow[t]{2}{*}{ 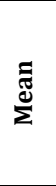 } & \multirow{2}{*}{ 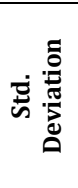 } & \multirow{2}{*}{ 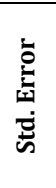 } & \multicolumn{2}{|c|}{$\begin{array}{c}95 \% \\
\text { Confidence } \\
\text { Interval for } \\
\text { Mean }\end{array}$} & \multirow{2}{*}{$\underset{\Xi}{\Xi}$} & \multirow{2}{*}{$\underset{\sum}{\stackrel{\Xi}{\Xi}}$} \\
\hline & & & & & $\begin{array}{l}\text { Lower } \\
\text { Bound }\end{array}$ & $\begin{array}{l}\text { Upper } \\
\text { Bound }\end{array}$ & & \\
\hline MS & 120 & 5.22 & 1.88 & 0.17 & 3.98 & 6.81 & 1.20 & 8.60 \\
\hline MHO & 120 & 4.45 & 1.46 & 0.14 & 3.50 & 5.68 & 0.80 & 9.50 \\
\hline Control & 120 & 1.84 & 0.77 & 0.05 & 1.24 & 2.32 & 1.00 & 3.20 \\
\hline
\end{tabular}

\begin{tabular}{|c|c|c|c|c|c|}
\hline $\begin{array}{c}\text { Source of } \\
\text { Variation }\end{array}$ & $\begin{array}{c}\text { Sum of } \\
\text { Squares }\end{array}$ & df & $\begin{array}{c}\text { Mean } \\
\text { Square }\end{array}$ & F & $\begin{array}{c}\text { p- } \\
\text { value }\end{array}$ \\
\hline Between Groups & 336.40 & 2 & 170.05 & \multirow{2}{*}{75.86} & \multirow{2}{*}{$0.0001, \mathrm{~S}$} \\
\hline Within Groups & 820.44 & 357 & 2.24 & & \\
\hline Total & $\mathbf{1 1 5 6 . 8 4}$ & $\mathbf{3 5 9}$ & & \\
\hline \multicolumn{6}{|c|}{ Table 3. One-Way ANOVA } \\
\hline
\end{tabular}

\begin{tabular}{|c|c|c|c|c|c|c|}
\hline \multirow{2}{*}{\multicolumn{2}{|c|}{ Group }} & \multirow{2}{*}{$\begin{array}{c}\text { Mean } \\
\text { Difference } \\
\text { (I-J) }\end{array}$} & \multirow{2}{*}{$\begin{array}{l}\text { Std. } \\
\text { Error }\end{array}$} & \multirow[b]{2}{*}{ p } & \multicolumn{2}{|c|}{$\begin{array}{c}\begin{array}{c}\text { 95\% Confidence } \\
\text { Interval }\end{array} \\
\end{array}$} \\
\hline & & & & & Lower & Upper \\
\hline MS & MHO & 0.67 & 0.19 & $0.002, \mathrm{~S}$ & 0.21 & 1.12 \\
\hline MHO & Control & 1.64 & 0.19 & $0.0001, \mathrm{~S}$ & 1.18 & 2.09 \\
\hline \multicolumn{7}{|c|}{ Table 4. Multiple Comparison- Tukey Test } \\
\hline
\end{tabular}




\begin{tabular}{|c|c|c|c|c|c|}
\hline Parameters & $\begin{array}{c}\text { MHO } \\
(n=120)\end{array}$ & $\begin{array}{l}\text { Control } \\
(n=120)\end{array}$ & p & $\begin{array}{l}\text { Odd's } \\
\text { Ratio }\end{array}$ & \begin{tabular}{|c}
$\begin{array}{c}95 \% \text { CI for } \\
\text { OR }\end{array}$ \\
\end{tabular} \\
\hline \multicolumn{6}{|c|}{ BMI $\left(\mathrm{Kg} / \mathrm{m}^{2}\right)$} \\
\hline Normal $(<25)$ & 0 & 120 & \multirow[b]{2}{*}{$0.0001, \mathrm{~S}$} & \multirow{2}{*}{0.000017} & \multirow{2}{*}{$\begin{array}{c}0.0000033- \\
0.00087\end{array}$} \\
\hline Obese ( $\geq 25)$ & 120 & 0 & & & \\
\hline \multicolumn{6}{|c|}{ Waist Circumference (Male) } \\
\hline $\operatorname{Normal}(<90 \mathrm{~cm})$ & 20 & 56 & \multirow{2}{*}{$0.0001, \mathrm{~S}$} & \multirow{2}{*}{0.022} & \multirow{2}{*}{$0.004-0.10$} \\
\hline Abnormal $(\geq 90 \mathrm{~cm})$ & 34 & 4 & & & \\
\hline \multicolumn{6}{|c|}{ Waist Circumference (Female) } \\
\hline Normal $(<80 \mathrm{~cm})$ & 24 & 56 & \multirow{2}{*}{$0.0001, \mathrm{~S}$} & \multirow{2}{*}{0.007} & \multirow{2}{*}{$\begin{array}{c}0.00043- \\
0.1215\end{array}$} \\
\hline Abnormal $(\geq 80 \mathrm{~cm})$ & 42 & 06 & & & \\
\hline \multicolumn{6}{|c|}{ Systolic Blood Pressure (mmHg) } \\
\hline Normal $(<130 \mathrm{mmHg})$ & 86 & 84 & \multirow{2}{*}{0.88 ,NS } & \multirow{2}{*}{0.92} & \multirow{2}{*}{$0.52-1.61$} \\
\hline Abnormal $(\geq 130 \mathrm{mmHg})$ & 34 & 36 & & & \\
\hline \multicolumn{6}{|c|}{ Diastolic Blood Pressure (mmHg) } \\
\hline Normal $(<85 \mathrm{mmHg})$ & 108 & 114 & \multirow{2}{*}{$0.19, \mathrm{NS}$} & \multirow{2}{*}{0.43} & \multirow{2}{*}{$0.14-1.28$} \\
\hline Abnormal ( $\geq 85 \mathrm{mmHg}$ ) & 10 & 6 & & & \\
\hline \multicolumn{6}{|c|}{ Fasting Blood Sugar (mg\%) } \\
\hline Normal $(<100 \mathrm{mg} / \mathrm{dL})$ & 102 & 117 & \multirow{2}{*}{$0.0001, \mathrm{~S}$} & \multirow{2}{*}{0.084} & \multirow{2}{*}{$0.019-0.37$} \\
\hline Abnormal $(\geq 100 \mathrm{mg} / \mathrm{dL})$ & 18 & 3 & & & \\
\hline \multicolumn{6}{|c|}{ HDL-Male } \\
\hline $\operatorname{Normal}(\geq 40 \mathrm{mg} / \mathrm{dL})$ & 24 & 38 & \multirow{2}{*}{$0.70, \mathrm{NS}$} & \multirow{2}{*}{0.85} & \multirow{2}{*}{$0.39-1.81$} \\
\hline Abnormal $(<40 \mathrm{mg} / \mathrm{dL})$ & 30 & 22 & & & \\
\hline \multicolumn{6}{|c|}{ HDL-Female } \\
\hline Normal $(\geq 50 \mathrm{mg} / \mathrm{dL})$ & 18 & 16 & \multirow{2}{*}{$0.41, \mathrm{NS}$} & & \\
\hline Abnormal $(<50 \mathrm{mg} / \mathrm{dL})$ & 48 & 44 & & 0.70 & $0.31-1.58$ \\
\hline & & riglycerides & & & \\
\hline Normal $(<150 \mathrm{mg} / \mathrm{dL})$ & 102 & 111 & 0 & 055 & 5 \\
\hline Abnormal ( $\geq 150 \mathrm{mg} / \mathrm{dL})$ & 18 & 09 & 0. & 0.55 & 5 \\
\hline & & CRP (mg/d) & & & \\
\hline Normal $(<3)$ & 36 & 108 & & & \\
\hline Abnormal $(\geq 3)$ & 84 & 12 & 0.0 & 0.0 & 11 \\
\hline ble 5 & 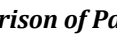 & & & & \\
\hline
\end{tabular}

\begin{tabular}{|c|c|c|c|c|c|}
\hline & Mean & $\begin{array}{c}\text { Std. } \\
\text { Deviation }\end{array}$ & $\mathbf{N}$ & $\begin{array}{c}\text { Correlation } \\
\text { 'r' }\end{array}$ & p-value \\
\hline hs-CRP & 4.45 & 1.46 & 120 & - & - \\
\hline BMI & 28.45 & 4.20 & 120 & 0.572 & $0.0001, \mathbf{S}$ \\
\hline WC & 93.14 & 15.48 & 120 & 0.432 & $0.0001, \mathbf{S}$ \\
\hline FBS & 92.56 & 16.56 & 120 & 0.114 & $0.214, \mathbf{N S}$ \\
\hline SBP & 126.41 & 10.66 & 120 & 0.054 & $0.560, \mathbf{N S}$ \\
\hline DBP & 86.66 & 6.72 & 120 & 0.006 & $0.945, \mathbf{N S}$ \\
\hline HDL & 34.75 & 12.21 & 120 & 0.165 & $0.072, \mathbf{N S}$ \\
\hline TG & 130.44 & 40.22 & 120 & 0.063 & $0.493, \mathbf{N S}$ \\
\hline
\end{tabular}

Table 6. Correlation between hs-CRP and Other Metabolic Variables in MHO Group. Pearson's Correlation Coefficient

By using Pearson's Correlation Coefficient significant positive correlation was found between hs-CRP BMI, WC.

\begin{tabular}{|c|c|c|c|c|c|c|c|c|c|}
\hline & \multicolumn{9}{|c|}{ Proportion of Elevated hs-CRP ( $\geq 3 \mathrm{mg} / \mathrm{L}$ ) } \\
\hline & \multicolumn{3}{|c|}{ Overall } & \multicolumn{3}{|c|}{ Males } & \multicolumn{3}{|c|}{ Females } \\
\hline & $\mathbf{n}$ & UOR & AOR & $\mathbf{N}$ & UOR & AOR & $\mathbf{n}$ & UOR & AOR \\
\hline $\begin{array}{l}\overrightarrow{0} \\
\text { 훙 }\end{array}$ & 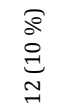 & - & -1 & 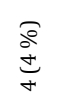 & -1 & - & $\begin{array}{l}\stackrel{5}{0} \\
\underbrace{0}_{\infty}\end{array}$ & - & $\neg$ \\
\hline$\stackrel{0}{\Sigma}$ & 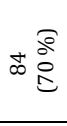 & 곡 & 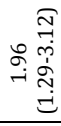 & 仿 & 空 & 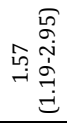 & 棺 & 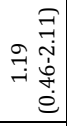 & 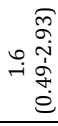 \\
\hline$\sum$ & 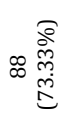 & & 舫 & 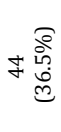 & 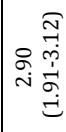 & 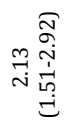 & 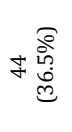 & 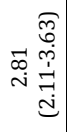 & Sু \\
\hline
\end{tabular}

Table 7. Proportion and Odds Ratios for hs-CRP across Weight and Metabolic Risk Factors Based on Phenotype

\section{DISCUSSION}

MS had the highest risk values followed by MHO and lastly the control group. The subclinical marker hs-CRP showed similar trends across the groups which were statistically significant $(\mathrm{p}<0.05)$. As these findings suggest, though MHO individuals had less than three metabolic risk parameters and decreased levels of all these risk markers for developing cardiovascular disease when compared with MS, still they had significantly higher values of these variable than controls. This also suggests that MHO may have an increased risk of progressing to MS in future. Studies have confirmed that MHO usually progresses to MS in future being a snap shot of an abnormal pre-metabolic state.[16,15] Justin B. Echouffo-Tcheugui et al. Examined Framingham Offspring Cohort, 4,291 participants who came for the examination cycles 2 to 7 and found out that the obesity sub-phenotypes progressed over time and MHO was the most short-term phenotype and a higher composite metabolic-BMI score was associated with a higher risk of a variety of clinical outcomes (diabetes, hypertension, cardiovascular events, and death) and high prevalence of subclinical cardiovascular disease cross-sectionally. An integrated assessment which accounted for shifts in the status of obesity sub-phenotypes over time were strongly associated with prevalent subclinical cardiovascular disease and clinical outcome incidence.[15]

It is needless to deny MHO as a pre-metabolic state and it would progress to overt MS with its consequent CVD risks in future. This study shows that hs-CRP in MHO and in the control-group was statistically significant and there was significant difference within and between groups suggesting that MHO portray trends of abnormal subclinical inflammation. The mean hs CRP in MS was $5.22 \pm 1.88$, in MHO was $4.45 \pm 1.46$ and in the control group it was $1.84 \pm 0.77$. The value was statistically significant across all the three groups. In one-way ANOVA there was significant difference within and between groups. Post hoc analysis with Tukey test showed MHO group had significantly increased value than control group. By using multiple regression analysis BMI, WC, were significantly associated with elevated hs-CRP [Table. 2]. An increased WC denotes visceral obesity which contributes to inflammation, insulin resistance, and fat deposition in liver. MHO population is vulnerable for all these abnormalities compared to MHNO individuals.[17]

In a study by Shaharyar S et al., the prevalence of abnormal hs-CRP values in MHO was $22 \%$ [18]. The overall AOR of high hs-CRP was 2.45 (males 2.51, females 3.59). The conclusion of this study was similar to the current study suggesting MHO as a non-healthy entity. In van Wijk DF et al., study there was a higher multivariable-adjusted hazard ratio for CHD in MHO subjects with CRP levels $>2 \mathrm{mg} / \mathrm{L}$. [19] The higher CRP levels were associated with a CHD risk. Iglesias Molli AE; in their study found that hs-CRP was significantly high in MHO than normal controls similar to the current study.[20] In our study proportion and Odd's ratios for hs-CRP across weight and metabolic risk factor based phenotype was assessed, the proportion of abnormal hs-CRP in MHO was $68.33 \%$, adjusted odd's (AOR) of abnormal hs-CRP in MHO was 1.9 times that of MHNO subjects. Bennett NR et al., analysed 342 men and 404 women with MHO.[21] Approximately $15 \%$ of the participants had high risk hs-CRP ( $>3 \mathrm{mg} / \mathrm{L})$, in logistic regression models high WC was associated with significantly higher odds of high hs-CRP (OR 7.8, 95\% CI 4.8-12.9, p<0.001) in MHO subjects.[21] An Indian study carried out by Acharya S et al; MHO population had increased mean HS-CRP levels $(4.01 \pm 1.68)$ as compared to normal controls $(2.16 \pm 0.56)$. Adjusted odd's ratio for high hs-CRP in MHO was 1.58 in males and 1.65 in females.[22] 


\section{Limitations}

This study is cross-sectional in design so it only throws light on associations of high risk subclinical pro-inflammatory markers with MHO phenotype and wouldn't prove the causation. This study defined MHO using a single criteria (NCEP ATP III).

\section{CONCLUSIONS}

Both MHO phenotype and MS are associated with higher affliction of inflammation when compared to MHNO subjects. This study proves that at any instance, asymptomatic MHO population have abnormal subclinical cardiovascular risk markers in a significantly increased proportion than a metabolic healthy non obese individual as abdominal obesity if accompanying with MHO further adds to the inflammatory cascade and risk of CVD. Time has come that we question the innocuous nature of $\mathrm{MHO}$ and its nomenclature should be changed to "Pre-Metabolic Syndrome" in the era of evidencebased medicine.

\section{REFERENCES}

[1] Puri R. Is it finally time to dispel the concept of metabolically-healthy obesity? Journal of the American College of Cardiology 2014;63 (24):2687-8.

[2] Norbert S, Hans-Ulrich H, Frank BH, et al. Metabolically healthy obesity: epidemiology, mechanisms and clinical implications. The Lancet Diabetes \& Endocrinology 2013;1 (2):152-62.

[3] Vliet-Ostaptchouk V, Nuotio ML, Slagter SN, et al. The prevalence of metabolic syndrome and metabolically healthy obesity in Europe: a collaborative analysis of ten large cohort studies. BMC Endocrine Disorders 2014;14 (1):9.

[4] Rey-López JP, de Rezende LF, Pastor-Valero M, et al. The prevalence of metabolically healthy obesity: a systematic review and critical evaluation of the definitions used. Obesity Reviews: an official journal of the International Association for the Study of Obesity 2014;15 (10):781-90.

[5] Matthias B. The distinction of metabolically 'healthy' from 'unhealthy' obese individuals. Current Opinion in Lipidology 2010;21 (1):38-43.

[6] Bell JA, Kivimaki M, Hamer M. Metabolically healthy obesity and risk of incident type 2 diabetes: a metaanalysis of prospective cohort studies. Obesity Reviews: An Official Journal of the International Association for the Study of Obesity 2014;15 (6):504-15.

[7] Roberson LL, Aneni EC, Wasim M, et al. Beyond BMI: the metabolically healthy obese phenotype \& its association with clinical/subclinical cardiovascular disease and allcause mortality- a systematic review. BMC Public Health 2014;14 (1):14.

[8] Yousuf O, Mohanty BD, Martin SS, et al. High-sensitivity Creactive protein and cardiovascular disease: a resolute belief or an elusive link? J Am Coll Cardiol 2013;62 (5):397-408.

[9] Misra A, Chowbey P, Makkar BM, et al. Consensus statement for diagnosis of obesity, abdominal obesity and the metabolic syndrome for Asian Indians and recommendations for physical activity, medical and surgical management. J Assoc Physicians India 2009;57:163-70.

[10] Geetha L, Deepa M, Anjana RM, et al. Prevalence and clinical profile of metabolic obesity and phenotypic obesity in Asian Indians. J Diabetes Sci Technol 2011;5 (2):439-46.

[11] Stefan N, Schick F, Häring HU. Causes, characteristics, and consequences of metabolically unhealthy normal weight in humans. Cell Metabolism 2017;26 (2):292-300.

[12] WHO STEP wise approach to surveillance (STEPS). Geneva, World Health Organization (WHO), 2008b.

[13] Pickering TG, Hall JE, Appel LJ, et al. Recommendations for blood pressure measurement in humans and experimental animals. Part 1: blood pressure measurement in humans: a statement for professionals from the Subcommittee of Professional and Public Education of the American Heart Association Council on High Blood Pressure Research. Hypertension 2005;45 (1):142-61.

[14] Roberts WL. CDC/AHA Workshop on Markers of Inflammation and Cardiovascular Disease: Application to Clinical and Public Health Practice: laboratory tests available to assess inflammation-performance and standardization: a background paper. Circulation 2004;110 (25):e572-6.

[15] Jung CH, Lee MJ, Hwang JY, et al. Association of metabolically healthy obesity with subclinical coronary atherosclerosis in a Korean population. Obesity (Silver Spring) 2014;22 (12):2613-20.

[16] Hinnouho GM, Czernichow S, Dugravot A, et al. Metabolically healthy obesity and the risk of cardiovascular disease and type 2 diabetes: The Whitehall II Cohort Study. Eur Heart J 2015;36 (9):551-9.

[17] Shaharyar S, Roberson LL, Jamal 0, et al. Obesity and metabolic phenotypes (metabolically healthy and unhealthy variants) are significantly associated with prevalence of elevated C-reactive protein and hepatic steatosis in a large healthy Brazilian population. Article ID 178526, Journal of Obesity 2015;2015:1-6.

[18] Echouffo-Tcheugui JB, Short MI, Xanthakis V, et al. Natural history of obesity sub-phenotypes: dynamic changes over two decades and prognosis in The Framingham Heart Study. The Journal of Clinical Endocrinology \& Metabolism 2019;104 (3):738-52.

[19] Van Wijk DF, Boekholdt SM, Arsenault BJ, et al. C-Reactive protein identifies low-risk metabolically healthy obese persons: The European Prospective Investigation of Cancer Norfolk Prospective Population Study. J Am Heart Assoc 2016;5 (6):e002823.

[20] Molli IAE, Steinhardt PA, López AP, et al. Metabolically healthy obese individuals present similar chronic inflammation level but less insulin-resistance than obese individuals with metabolic syndrome. PLoS One 2017;12 (12): 0190528.

[21] Bennett NR, Ferguson TS, Bennett FI, et al. Highsensitivity C-reactive protein is related to central obesity and the number of metabolic syndrome components in Jamaican young adults. Front Cardiovasc Med 2014;1:12.

[22] Acharya S, Shukla S, Wanjari A. Subclinical risk markers for Cardiovascular Disease (CVD) in Metabolically Healthy Obese (MHO) subjects. Journal of Clinical and Diagnostic Research 2019;13 (6):0C01-0C06. 